\title{
IGCP Projects 2008
}

(OET - on extended term)

No. 475 Deltas in the Monsoon AsiaPacific Region (DeltaMAP)

Project leaders: S. Goodbred, Jr. (USA), Y. Saito (Japan)

Duration: 2003-2007 (OET)

Website: http://unit.aist.go.jp/igg/rg/cugrg/ADP/ADP_E/a_igcp4751_en.html

No. 478 Neoproterozoic-Early Palaeozoic Events in South-West-Gondwana Project leaders: C. Gaucher (Uruguay), P. C. Boggiani (Brazil), A. Braun (Germany), H. Hartwig Frimmel (Germany), J.B. Germs (South Africa), D. G. Poiré (Argentina)

Duration: 2003-2007 (OET)

Official website: www.igcp478.com

Mirror website:

www.vssagi.com/igcp478/igcp478.htm

No. 480 Tectonics of Central Asia

Project leaders:B. Natal'in (Turkey), A. Yin (USA), A. M. C. Sengör (Turkey), M.

Kuzmin (Russia), Shuwen Dong (China)

Duration: 2005-2009

Website: http://www.igcp.itu.edu.tr/

\section{No. 481 Dating Caspian Sea Level}

\section{Change}

Project leaders: S.B. Kroonenberg (Netherlands), S. Leroy (UK)

Duration: 2003-2007 (OET)

Website: http://www.caspage.citg.tudelft.nl www.caspiansealevelchange.org http://black.sealevel.ca

\section{No. 486 Au-Ag-Telluride-Selenide}

Deposits

Project leaders: N. J. Cook (Norway), K. Kojonen (Finland)

Duration: 2003-2007 (OET)

Website: www.ngu.no/igcp486

No. 487 Seismic Microzoning of Latin American Cities

Project leaders: J. L. Alvarez Gómez (Cuba), A. A. Giesecke Matto (Peru), G. F. Panza (Italy)

Duration: 2004-2008

Website: www.ictp.trieste.it/ sand/SMLAC /SMLAC.html

\section{No. 493 The Rise and Fall of the}

Vendian Biota

Project leaders: M. Fedonkin (Russia), P. Vickers-Rich (Australia), J. Gehling (Australia)

Duration: 2003-2007 (OET)

Website: http://www.geosci.monash.edu.au /precsite
No. 495 Quaternary Land-Ocean Interactions

Project leaders: A. Long (UK), S. Islam (Bangladesh)

Duration: 2004-2008

Website: www.geography.dur.ac.uk/projects/igcp495

\section{No. 497 The Rheic Ocean}

Project leaders: U. Linnemann (Germany),

R. D. Nance (USA), M. de Wit (South

Africa), E. Bozkurt (Turkey), P. Kraft

(Czech Republic), F. Pereira (Portugal),

R. A. Strachan (UK)

Duration: 2004-2008

Website: http://www.snsd.de/igcp497/

No. 499 Devonian Land-Sea Interaction: Evolution of Ecosystems and Climate in the Devonian

Project leaders: P. Königshof (Germany), J. Lazauskiene (Lithuania), E. Schindler

(Germany), Volker Wilde (Germany) and

N. Yalçin (Turkey)

Duration: 2004-2008

Website: http://www.senckenberg.de/igcp499

No. 500 Dryland Change: Past, Present, Future

Project leader: D.Thomas (UK)

Duration: 2004-2008

Website: http://igcp500.ouce.ox.ac.uk/

No. 502 Global Comparison of Volcanichosted Massive Sulphide Districts Project leaders: R.Allen (Sweden), F. Tornos (Spain), J. Peter (Canada), N. Çagatay (Turkey)

Duration: 2004-2008

Website: http://www.1tu.se/tkg/

http://www.1tu.se/tkg/avd/kgo/forsk/IGCP

No. 503 Ordovician Palaeogeography and Palaeoclimate

Project leaders: T. Servais (France), D.A.T.

Harper (Denmark), J. Li (China), A.

Munnecke (Germany), A. W. Owen

(UK), P.M. Sheehan (USA)

Duration: 2004-2008

Website: http://sarv.gi.ee/igcp503/

No. 506 Marine and Non-marine Jurassic

Project leaders: Jingeng Sha (China), Nicol Morton (France), W. A.P. Wimbledon (UK), Paul E. Olsen (USA), Alberto G. Riccardi (Argentina), Grzegorz (Gregory) Pieñkowski (Poland), Yongdong Wang (China)

Duration: 2005-2006 (2009)

Website: http://www.nigpas.ac.cn/IGCP506 index.asp
No. 507 Paleoclimates of the Cretaceous in Asia

Project leaders: Yong Il Lee (Korea), Xiaoqiao Wan (China), Takashi Sakai (Japan), and Krishnan Ayyasami (India)

Duration: 2006-2010

Website: http://igcp507.kopri.re.kr/

No. 509 Palaeoproterozoic Supercontinents and Global Evolution

Project leaders: S.M. Reddy (Australia), D. Evans (USA), R. Mazumder (India)

Duration: 2005-2009

Website:

http://earth.geology.yale.edu/igcp509/

No. 510 A-type Granites and Related Rock through Time

Project leaders: Roberto Dall'Agnol (Brazil), Carol D. Frost (USA), O. Tapani

Rämö (Finland), L.J. Robb (South Africa) Duration: 2005-2009

Website: http://www.igcp-510.org

No. 511 Submarine Mass Movements and their Consequences

Project leaders: Jacques Locat (Canada), Juergen Mienert (Norway) and Roger Urgeles (Spain)- (IOC link)

Duration: 2005-2009

Website: http://www.geohazards.no/IGCP511

No. 512 Neoproterozoic Ice Ages

Project leaders: Graham A. Shields (Aus-

tralia), Emmanuelle Arnaud (Canada)

Duration: 2005-2009

Website: www.IGCP512.org

No. 513 Karst Aquifers and Water Resources

Project leaders: Chris Groves (USA), Yuan Daoxian (China), Bartolome AndreoNovarro (Spain), Heather Viles (UK) Duration: 2005-2009

Website: http://hoffman.wku.edu/igcp/513.h tml (General information)

http://hoffman.wku.edu/karst2007/k2007.html http://www.wku.edu/cehp http://www.cosis.net/members/meetings/s essions/information.php?p_id=247\&s_id $=4433$

No. 514 Fluvial Palaeosystems: Evolution and Mineral Deposits

Project leaders: N. Patyk-Kara (Russia), A. Duk-Rodkin (Canada), Baohong Hou (Australia), Li Ziying (China), Vladimir Dolgopolov (Kazakhstan)

Duration: 2005-2009

Website: http://www.igem.ru/igcp514/

No. 516 Geological Anatomy of East and South East Asia

Project leaders: Ken-ichiro Hisada (Japan), Punya Charusiri (Thailand), Byung-Joo Lee (Rep. of Korea), Xiaochi Jin (China) Duration: 2005-2009

Website: http://staff.aist.go.jp/harahide/igcp516 
No. 519 Hydrogeology, Hydrochemistry and Management of Coastal Aquifers on the Atlantic Coast of South America

Project leaders: Emilia Bocanegra (Argentina), Emilio Custodio (Spain), Marisol Manzano (Spain), Gerson Cardoso (Brazil), Jenny Reynolds Vargas (Costa Rica)

Duration: 2005-2009

Website:

http://www.mdp.edu.ar/exactas/geologia/cgcyc/hidrogeologia.html

No. 521 Black sea Mediterranean Corridor during the last $30 \mathrm{ky}$ : Sea level change and human adaptation

Project leaders: Valentina Yanko-Hombach (Canada), Yucel Yilmaz (Turkey), Pavel Dolukhanov (UK)

Duration: 2005-2009

Website: http://www.avalon-institute.org/IGCP http://black.sealevel.ca

http://www.bridge.bris.ac.uk/projects/EM BSECBIO

http://www.paleontol.geo.sfedu.ru

\section{No. 523 GROWNET - Gobal Ground}

Water Network

Project leaders: Shrikant Daji Limaye (India), Antony J Reedman (UK)

Duration: 2005-2009

Website: http://www.igcp-grownet.org

No. 524 Arc-Continent Collision

Project leaders: Denis Brown (Spain), ChiYue Huang (Taiwan)

Duration: 2007-2009

Website: www.ija.csic.es/gt/IGCP524

No. 526 Risks Resources and Record of the Past on the Continental Shelf

Project leaders: Francesco L.atino Chiocci (Italy), Lindsay Collins (Australia), Michel Michaelovitch de Mahiques (Brazil), Renée Hetherington (Canada)

Duration: 2007-2011

No. 529 Availability of groundwater resources in selected urban areas in Southern African Development Community (SADC) region

Project leaders: Imasiku A. Nyambe (Zambia)

Duration: 2007-2011

No. 540 Gold-bearing hydrothermal fluids of oregenic deposits

Project leaders: P.S. Garofalo (Italy), J.R.

Ridley (USA), Vsevolod Prokof'ev (Russia)

Duration: 2007-2011

Website: http://www.geomin.unibo.it/igcp_540

No. 543 Low-temperature ther-

mochronology: applications and interlaboratory calibration

Project leaders: Massimiliano Zattin (Italy), J. I. Garver (USA), Vitaliy A. Privalov (Ukraine), Alexei V. Soloviev (Russia),

Cornelia Spiegel (Germany), Maarten de
Wit (South Africa), Dewen Zheng (China)

Duration: 2007-2010

No. 545 Clays and clay minerals in

Africa

Project leaders: Georges-Ivo E. Ekosse (South Africa)

Duration: 2007-2011

Website: http://www.saweb.co.za/claymineralsafrica/

No. 546 Subduction zones of the

Caribbean

Project leaders: Antonio Garcia-Casco (Spain), Uwe Martens (USA)

Duration: 2007-2011

Website: http://www.ugr.es/ agcasco/igcp546/

No. 555 Rapid Environmental/Climate

Change in the Cretaceous Greenhouse

World

Project leaders: Chengshan Wang (China), Robert Scott (USA), Hugh Jenkyns (UK), Michael Wagreich (Austria), William

Hay (USA); Zakharov Y.D. (Russia)

Duration: 2007-2010

Website: www.cretaceousworld.com/igcp555

No. 557 Diamonds, xenoliths and kim-

berlites

Project leaders: Holger Sommer

(Botswana), Klaus Regenauer-Lieb (Australia), Christoph Hauzenberger (Austria) Jonathan Kashabano (Tanzania), Gétan Moloto-A-Kenguemba (Central African Republic)

Duration: 2007-2011

Website: http://igcp557.uni-graz.at/

No. 559 Crustal Architecture and landscape Evolution

Project leaders: Bruce R. Goleby (Australia) and 14 members (USA, Canada, China, Finland, Netherlands, New

Zealand, Russia)

Duration: 2008-2012

No. 565 Geodetic Monitoring of the

Global Water Cycle

Project leaders: Hans-Peter Plag (USA), Richard S. Gross (USA), Markus Rothacher (Germany), Norman L. Miller (USA), Susanna Zerbini (Italy), Chris Rizos (Australia)

Duration: 2008-2012

No. 567 Earthquake Archaeology -Archaeoseismology along the Alpine-

Himalayan seismic zone

Project leaders: Manuel Sintubin (Belgium), Iain Stewart (UK), Tina Niemi (USA), Erhan Altunel (Turkey)

Duration: 2008-2012

No. 572 Permian-Triassic Ecosystems

Project leaders: Zhong Qiang Chen (Australia), Richard J. Twitchett (UK), Jinnan Tong (China), Margret L. Fraiser (USA), Sylvie Crasquin (France), Steve Kershaw
(UK), Thomas J. Algeo (USA), Kliti

Grice (Australia)

Duration: 2008-2012

Funded projects

O.E.T.

Total

\section{IGCP Secretariat}

UNESCO, Division of Ecological and Earth Sciences

1, Rue Miollis

75732 Paris Cedex 15

FRANCE

Tel: +33145684118

Fax: +33145685822

www.unesco.org/sciencelearth

E-mail:igcp@unesco.org 